\title{
Anti-inflammatory and pro-angiogenic effects of beta blockers in a canine model of chronic ischemic cardiomyopathy: comparison between carvedilol and metoprolol
}

\author{
D. Elizabeth Le, \\ Hospital and Specialty Medicine - Cardiology, Portland VA Medical Center and Knight \\ Cardiovascular Institute, Portland, OR, USA; Knight Cardiovascular Institute, OHSU, UHN62, \\ 3181 SW Sam Jackson Park Road, Portland, OR 97239, USA \\ Marco Pascotto, \\ Buon Consiglio-Fatebenefratelli Hospital, Via Posillipo 42, 80123 Naples, Italy \\ Howard Leong-Poi, \\ Heart and Stroke Foundation of Canada and the Canadian Institutes of Health Research, Ottawa, \\ Canada \\ Ibrahim Sari, \\ The Turkish Cardiac Society, Istanbul, Turkey \\ Antonio Micari, and \\ Italian Society of Cardiology-Aventis Foundation, Milan, Italy \\ Sanjiv Kaul \\ Knight Cardiovascular Institute, OHSU, UHN62, 3181 SW Sam Jackson Park Road, Portland, OR \\ 97239, USA
}

\begin{abstract}
There is controversy regarding the superiority of carvedilol (C) over metoprolol (M) in congestive heart failure. We hypothesized that $\mathrm{C}$ is superior to $\mathrm{M}$ in chronic ischemic cardiomyopathy because of its better anti-inflammatory and pro-angiogenic effects. In order to test our hypothesis we used a chronic canine model of multivessel ischemic cardiomyopathy where myocardial microcatheters were placed from which interstitial fluid was collected over time to measure leukocyte count and cytokine levels. After development of left ventricular dysfunction, the animals were randomized into four groups: sham $(n=7)$, placebo $(n=8), \mathrm{M}(n=11)$, and $\mathrm{C}(n=$ 10 ), and followed for 3 months after treatment initiation. Tissue was examined for immunohistochemistry, oxidative stress, and capillary density. At 3 months both rest and stress wall thickening were better in $\mathrm{C}$ compared to the other groups. At the end of 3 months of treatment endsystolic wall stress also decreased the most in C. Similarly resting myocardial blood flow (MBF) improved the most in $\mathrm{C}$ as did the stress endocardial/epicardial MBF. Myocardial interstitial fluid showed greater attenuation of leukocytosis with $\mathrm{C}$ compared to $\mathrm{M}$, which was associated with less fibrosis and oxidative stress. C also had higher IL-10 level and capillary
\end{abstract}

(C) Springer-Verlag Berlin Heidelberg 2013

S. Kaulkauls@ohsu.edu.

Present Address: H. Leong-Poi St, Michael's Hospital, 30 Bond Street, Toronto, ON M5B 1W8, Canada

Present Address: I. Sari Department of Cardiology, Gaziantep University, 27310 Gaziantep, Turkey

Present Address: A. Micari GVM Care and Research Maria Eleonora Hospital, Viale Regione Siciliana, 1571 Palermo, Italy

Presented in part at the 57th annual scientific sessions of the American College of Cardiology in Chicago, Illinois, March 2008.

Conflicts of interest None. 
density. In conclusion, in a chronic canine model of multivessel ischemic cardiomyopathy we found 3 months of $\mathrm{C}$ treatment resulted in better resting global and regional function as well as better regional function at stress compared to $\mathrm{M}$. These changes were associated with higher myocardial levels of the anti-inflammatory cytokine IL-10 and less myocardial oxidative stress, leukocytosis, and fibrosis. Capillary density and MBF were almost normalized. Thus in the doses used in this study, $\mathrm{C}$ appears to be superior to $\mathrm{M}$ in a chronic canine model of ischemic cardiomyopathy from beneficial effects on inflammation and angiogenesis. Further studies are required for comparing additional doses of these drugs.

\section{Keywords}

Ischemic cardiomyopathy; Beta blockers; Regional flow; Regional function; Cytokines; Angiogenesis

\section{Introduction}

Based on the results of large clinical trials $\beta$-adrenergic receptor blockers (BB) have become the mainstay of therapy in congestive heart failure (CHF) $[27,33,36]$. Whereas previously they were thought to exert their beneficial effects solely through anti-adrenergic or antiischemic $[13,20]$ mechanisms, it has now become increasingly clear that they also have important anti-inflammatory properties, which can offer independent salutary effects in CHF [24]. Even though it has been reported that carvedilol (a non-selective BB with vasodilatory and antioxidant effects) is superior to metoprolol (a selective $\beta 1$ blocker) in patients with CHF $[36,40]$ this finding remains controversial [7, 25].

Almost all clinical trials evaluating BB have included patients with both ischemic and nonischemic cardiomyopathy. Furthermore, a sizeable proportion of patients with ischemic cardiomyopathy have had prior myocardial infarction (MI). Previous animal studies of BB in ischemia have mostly utilized infarct models where the myocardial effects of BB have been studied at one point in time at autopsy [21,30, 31,37]. Thus, the time course of BB effects in CHF has not been studied in animals. Furthermore, a large number of patients with ischemic cardiomyopathy do not have a prior MI. These patients either have repetitive stunning (severe coronary stenosis with reduced coronary flow reserve leading to repeated episodes of myocardial ischemia during stress) or hibernation [reduced resting myocardial blood flow (MBF) from severe coronary narrowing] $[5,6,8,14,15]$. There has been no investigation on the regional myocardial effects of $\mathrm{BB}$ in an animal model that mimics one or both of these two latter situations.

In the present study, we hypothesized that BB have a beneficial role in a canine model of chronic ischemic cardiomyopathy through their anti-inflammatory and proangiogenic effects and carvedilol may be superior to metoprolol in this regard. In order to test our hypothesis we gave BB therapy to dogs with chronic ischemic cardiomyopathy produced by placing ameroid constrictors on the proximal portions of the left anterior descending (LAD) and left circumflex (LCx) coronary arteries and their major branches [the right coronary artery does not supply the left ventricular (LV) myocardium in the dog]. Over time the constrictors occlude the coronary arteries producing chronically ischemic myocardial segments with either stunning or hibernation, but no necrosis. We have described the features of this model previously $[2,3,11,34]$, and have tested it for various therapies aimed at reducing chronic myocardial ischemia and CHF $[18,19]$.

For this study, animals were chronically instrumented to measure left atrial and aortic pressures and radiolabeled microsphere-derived MBF. Regional LV systolic function was 
measured at regular intervals using 2-dimensional echocardiography (2DE). In addition, microcatheters were placed within the myocardium from which interstitial fluid could be collected periodically over time to measure leukocyte count and cytokine levels that reflect myocardial inflammation.

\section{Methods}

\section{Animal preparation}

The study was approved by the Animal Research Committee and conformed to the American Heart Association Guidelines for the Use of Animals in Research. Forty-three adult mongrel dogs (weight $=30-35 \mathrm{~kg}$ ) were used. Of these, seven underwent sham surgery (dissection of coronary arteries and insertion of microcatheters only) and the remaining 36 animals were instrumented to create chronic ischemic cardiomyopathy. They were given 75 $\mathrm{mg}$ of aspirin daily for 3 days prior to surgery and Gentamicin $(80 \mathrm{mg}$ ) and Cefazolin $(1 \mathrm{~g})$ immediately prior to surgery, and Cefazolin alone daily through post-operative day 3 .

Surgery was performed under sterile conditions. Anesthesia was induced with $20 \mu \mathrm{g} \mathrm{kg}^{-1}$ of fentanyl (Abbott Laboratories, Chicago, IL), $400 \mu \mathrm{g} \mathrm{kg}^{-1}$ of etomidate (Bedford Laboratories, Bedford, Ohio), and $300 \mu \mathrm{g} \mathrm{kg}^{-1}$ of diazepam (Elkins-Sinn Inc., Cherry Hill, $\mathrm{NJ})$ administered intravenously. The animals were intubated and anesthesia was maintained with a mixture of 1-1.5\% isoflurane, $\mathrm{O}_{2}$, and air given through a respirator (Model 607, Harvard Apparatus, South Natick, MA). Minute volume was set between 5.5 and $6.5 \mathrm{~L}$ $\mathrm{min}^{-1}$ to maintain a physiologic $\mathrm{PCO}_{2}$. Heart rate and electrocardiogram were monitored throughout the operation.

A small incision was made in the right groin and a $6 \mathrm{~F}$ indwelling catheter (Cook Instruments, Bloomington, IN) was inserted into the femoral artery and secured in place with silk ties. The catheter was flushed with a dilute solution of heparin (Sololak Laboratories, Elk Grove Village, IL) and capped off with a rubber injection port. It was then tunneled beneath the skin in the groin to allow subsequent transcutaneous access for arterial pressure monitoring, as well as withdrawal of samples for blood gas and radiolabeled microsphere-derived MBF analyses. The groin incision was then closed in layers.

A left lateral thoracotomy was performed in the fourth intercostal space and the heart was suspended in a pericardial cradle. The proximal portions of the LAD and LCx were dissected free from surrounding tissues, and any large proximal branches of these arteries were similarly dissected. Three or more appropriately sized $(1-3.5 \mathrm{~mm})$ ameroid constrictors (Medical Research \& Manufacturing, Corvallis, OR) were placed around these arteries. LV function was assessed by epicardial $2 \mathrm{DE}$ after placement of each ameroid constrictor to ensure that no deterioration in regional systolic function occurred.

Four microcatheters (two in the LAD and 2 in the LCx regions) were inserted in the myocardium at the low-papillary muscle level using a 14-gauge catheter. They were threaded through approximately $3 \mathrm{~cm}$ of myocardium before retrieving them again on the epicardial surface of the heart. In this manner, a portion of the catheter containing multiports (see below) was embedded in the myocardium with the two ends extruding out. Epicardial 2DE was performed to confirm that the entire lengths of the catheters were within the myocardium without entering the LV cavity. Both free ends of the microcatheters were then tunneled beneath the dorsal skin to allow subsequent access for myocardial interstitial fluid collection.

A $6 \mathrm{~F}$ indwelling catheter was inserted in the left atrium and secured in placed with prolene sutures. After flushing with a dilute heparin solution, its end was capped off with a rubber 
injection port and buried beneath the abdominal skin to allow subsequent transcutaneous injections of radiolabeled microspheres. The chest was closed in layers, and the animal was revived and transferred to a heated and oxygenated observation area. Pain medications were administered as needed. The animals were examined at least twice a day for the follow-up period of 6 months and treated for heart failure (as evidenced by tachypnea, poor appetite, and reluctance to engage with handlers) or infection, if required.

\section{Microcatheter preparation}

Microcatheters were constructed by creating 40 multiports in a $1 \mathrm{~cm}$ segment of a $120 \mathrm{~cm}$ Micro-Renathane catheter $(0.04 \mathrm{~mm}$ inner diameter; $0.8 \mathrm{~mm}$ outer diameter; Braintree Scientific Inc, Braintree, MA), using a 25 -gauge needle. A $0.5 \mathrm{~cm} \times 0.5 \mathrm{~cm}$ gauze piece was then sutured to the catheter using 4-0 prolene and was secured in place using medical adhesive silicone type A (Dow Corning Corp, Midland, MI). The gauze was used to anchor the catheter to the epicardium at the time of insertion into the myocardium. The microcatheters were gas sterilized.

\section{Myocardial interstitial fluid collection}

Lactated Ringer's solution was infused from one end of the microcatheter at $10 \mu \mathrm{L} \mathrm{min}^{-1}$ using a constant rate pump (Model 944, Harvard Apparatus) and the fluid from the other end was collected in Eppendorf tubes on ice. Interstitial fluid was collected in two aliquots of $100 \mu \mathrm{L}$ each (equal to the dead space of the catheter) and discarded. Then $1 \mathrm{~mL}$ of fluid was collected for leukocyte count followed by five aliquots of $100 \mu \mathrm{L}$ each for cytokine level measurement. Fluid for measuring the leukocyte count was transported on ice to the clinical hematology laboratory and the five aliquots for cytokine level measurement were stored at $-80{ }^{\circ} \mathrm{C}$.

\section{Myocardial interstitial fluid analysis}

Interstitial fluid leukocyte count was measured using an automated analyzer (Sysmex XE-2100, Kobe, Japan) and then confirmed using a hemocytometer. The Bio-Plex suspension array system was used to quantify cytokine levels from myocardial interstitial fluid and plasma collected from venous blood. Levels (in $\rho g \mathrm{~mL}^{-1}$ ) of TNF- $\mathrm{a}$, IL-6, IL-1 $\beta$, and IL-10 were measured (Bio-Rad Laboratories, Inc, Hercules, CA). The samples were incubated with antibody-coupled beads, washed, incubated with biotinylated detection antibody, washed again, and then incubated with streptavidin-phycoerythrin and read on a microplate-based bioassay system. The inter-assay variation was $<30 \%$ and intra-assay variation was $<20 \%$. In order to maximize the accuracy, all samples were measured in duplicate at the same time.

\section{Myocardial blood flow measurements}

Because the duration of the protocol was 5-6 months we used radiolabeled microspheres with long half-lives and adequate separation of the energy windows to measure regional MBF. In each dog four of the following five [with their energy windows ( $\mathrm{keV})$, and halflives (days)] microspheres were used: ${ }^{141} \mathrm{Ce}, 120-175,33 ;{ }^{113} \mathrm{Sn}, 340-440,115 ;{ }^{103} \mathrm{Ru}$, $450-550,39 ;{ }^{95} \mathrm{Nb}, 680-840,35$; and ${ }^{46} \mathrm{Sc}, 842-1,300,84$. The microspheres with longer half-lives were injected earlier in the protocol to allow enough counts in the sample on postmortem analysis. Approximately $2 \times 10^{6} 11 \mu \mathrm{m}$ radiolabeled microspheres (Dupont Medical Products, Wilmington, DE) were suspended in $4 \mathrm{~mL}$ of normal saline- $0.01 \%$ tween 80 solution and injected into the left atrium over $20 \mathrm{~s}$. This dose of radiolabeled microspheres allows at least 1,000 microspheres to be counted in each gram of normal tissue and at least 300 microspheres in each gram of ischemic tissue. Reference samples were withdrawn from the femoral artery over $130 \mathrm{~s}$ with a constant rate withdrawal pump (Harvard Apparatus, 
Model 944). The post-mortem heart slices (see later), corresponding to the mid-papillary muscle 2DE short-axis image, were cut into 16 wedge-shaped pieces. Each piece was further divided into epicardial, mid-myocardial, and endocardial portions.

The tissue and arterial reference samples were counted in a gamma well counter with a multichannel analyzer (Model 1282, LKB Wallac, Washington DC). Corrections were made for activity spill-over from one window to the next using a set of simultaneous equations. MBF to each sample was calculated by the equation $Q_{\mathrm{m}}=\left(C_{\mathrm{m}} \times Q_{\mathrm{r}}\right) / \mathrm{C}_{\mathrm{r}}$ where $Q_{\mathrm{m}}$ flow $(\mathrm{mL}$ $\left.\min ^{-1}\right), C_{\mathrm{m}}$ tissue counts, $Q_{\mathrm{r}}$ rate of arterial blood withdrawal $\left(\mathrm{mL} \min ^{-1}\right)$, and $C_{\mathrm{r}}$ counts in the reference sample [16]. Transmural MBF $\left(\mathrm{mL} \mathrm{min}^{-1} \mathrm{~g}^{-1}\right)$ to each segment was derived by dividing the sum of MBF to individual segments by their combined weight. Transmural MBF for the LCx and LAD beds was calculated by averaging the transmural MBF in the segments within the central $75 \%$ of each bed. Average endocardial and epicardial MBF were similarly calculated and the endocardial/epicardial MBF ratio was derived.

\section{Hemodynamic data}

For arterial pressure measurements, tubing primed with heparinized normal saline was connected at one end to a pressure transducer (model 1295A, Hewlett Packard, Palo Alto, $\mathrm{CA}$ ) and at the other end to the arterial catheter. This transducer and the electrocardiographic port were connected to a multi-channel recorder (model 4568C, Hewlett-Packard), which in turn was connected to a computer. The signals were displayed online using Labtech Notebook (Laboratory Technologies Corp., Wilmington, MA) and were digitally acquired for later analysis.

\section{Two-dimensional echocardiography}

2DE was performed with a phased-array system (Sonos 7500, Philips Ultrasound, Andover, MA) using a 2.3 MHz probe. Apart from the intraoperative 2DE described above, all data were acquired with the dog lying on its left side and imaging performed from the right thorax. Although the examination was performed using multiple views, only three short-axis views were recorded on each examination. Care was taken to acquire the same views every time in an individual dog.

For each animal, the short-axis view displaying the maximal degree of global LV dysfunction at any time during follow-up was identified. All wall thickening (WT) measurements were then made at this single level (usually the mid-papillary muscle). An entire systolic contraction sequence (from end-diastole to end-systole) was selected from each examination and the images were transferred to a custom-designed offline image analysis system.

Our method for measuring regional \%WT has been described previously [42]. In brief, 8-12 epicardial and endocardial targets were defined by the observer in each frame from enddiastole to end-systole. These points were then automatically connected using cubic-spline interpolation to derive the epicardial and endocardial contours. In order to correct for cardiac systolic rotation, the junction of the posterior LV free wall and the right ventricular free wall was defined over the epicardium in each frame. The computer generated 100 equidistant chords between the two contours starting at this point with each chord representing the shortest distance between the epicardial and endocardial contours. The observer then identified the regions of the myocardium in which the chord lengths were averaged in each frame. Plots of \%WT over the entire systolic contraction sequence in the central $75 \%$ of these regions were then generated, with time represented in deciles. 
End-systolic wall stress was calculated using the modified formula: Wall stress $\left(\mathrm{g} \mathrm{cm}^{-2}\right)=$ $[(1.35)($ mean arterial pressure $) \times(\mathrm{LV}$ diameter at end-systole $)] /[(4) \times($ wall thickness at endsystole $) \times(1+$ wall thickness at end-systole/LV diameter at end-systole $)][39]$.

\section{Gross and microscopic histology and immunohistochemistry}

The heart slice corresponding to the 2DE image was immersed in a solution of 1.3\% 2,3,5 triphenyl tetrazolium chloride (TTC) (Sigma Corp., St Louis, MO) and 0.2 M Sörensen's buffer $\left(\mathrm{KH}_{2} \mathrm{PO}_{4}\right.$ and $\mathrm{K}_{2} \mathrm{HPO}_{4}$ in distilled water, $\left.\mathrm{pH} 7.4\right)$ at $37{ }^{\circ} \mathrm{C}$ for $20 \mathrm{~min}$. Using this method, areas of viable myocardium stain brick red whereas infarcted areas do not take up the stain due to lack of enzymatic activity [12]. All analysis was performed by observers blinded to the source of tissue.

Myocardial tissue was fixed in formalin and paraffin-embedded for histologic analysis. Fibrosis was identified by staining deparaffinized tissue sections ( $5 \mu \mathrm{m}$ thick) for connective tissue using Masson's trichrome. ImageJ v146r (open source software, http:// rsb.info.nih.gov/ij) was used to quantify the amount of fibrosis at $10 \times$ magnification and was expressed as area of fibrosis/total area of tissue $\times 100$.

Endothelial cells of capillaries were identified using immunohistochemical staining with the antibody to von Willebrand factor. Deparaffinized tissue sections ( $5 \mu \mathrm{m}$ thick) were pretreated with proteinase $\mathrm{K}, 20 \mu \mathrm{g}(20 \mathrm{~min})$ and $3 \% \mathrm{H}_{2} \mathrm{O}_{2}$ in PBS (15 min). All rinses were with tris-buffered saline (TBS), $\mathrm{pH} 7.6$ with $0.05 \%$ Tween-20. Sections were incubated with $3 \%$ normal goat serum in phosphate-buffered saline (blocking serum) for 20 $\mathrm{min}$ at room temperature, followed by an avidin/biotin blocking step. Sections were incubated with the primary antibody (Dako polyclonal rabbit anti-human von Willebrand factor, code A0082) diluted 1:1,600 in the blocking serum, overnight at $4{ }^{\circ} \mathrm{C}$. The secondary antibody, biotinylated goat anti-rabbit (diluted 1:200 in blocking serum, Vector), was applied to the tissue for $60 \mathrm{~min}$ at room temperature, and sections were incubated with avidin-biotin-peroxidase complex (Vectastain Elite kit, Vector) for $30 \mathrm{~min}$. The color reaction was visualized with diaminobenzidine (DAB). The number of capillaries was manually counted in two representative fields at $40 \times$ magnification and averaged. Capillary density was expressed as the number of capillaries per high power field. In sections with fibrosis, a representative area immediately adjacent to the fibrotic tissue was selected for analysis.

Apoptotic myocytes were stained using the ApopTag® Peroxidase In Situ Apoptosis Detection Kit (EMD Millipore Corporation, Billerica, Massachusetts, USA). Deparaffinized tissue sections ( $5 \mu \mathrm{m}$ thick) were stained according to kit instructions. The number of apoptotic myocytes was manually counted in 3 representative fields at $40 \times$ magnification and averaged. The density of apoptotic myocytes was expressed as the number of apoptotic myocytes per high power field.

\section{Oxidative stress analysis}

Frozen myocardial tissue was homogenized and supernatant was collected. Using a commercial kit (Biovision, Inc, Milpitas, CA), malondialdehyde (MDA), a product of lipid peroxidation, was quantified using a colorimetric assay and expressed as $\mathrm{nM} \mathrm{mg}$ of protein $^{-1}[29]$.

Total isoprostanes III and VI (IsoP III, IsoP VI), products of arachidonic acid peroxidation, were also measured using liquid chromatography-tandem mass spectrometry. Isoprostanes were resolved on a Phenomenex Hyperclone BDS C18 $130 \AA 150 \times 2 \mathrm{~mm}, 3 \mu \mathrm{m}$ column kept at $40{ }^{\circ} \mathrm{C}$ using a Shimadzu CTO-20AC column oven. The high-performance liquid chromatography (HPLC) system consisted of a Shimadzu SIL-20AC XR auto-sampler, 2 
LC-20AD XR LC pumps. The gradient mobile phase was delivered at a flow rate of $0.4 \mathrm{~mL}$ $\min ^{-1}$, and consisted of two solvents, A: $0.005 \%$ acetic acid in water and B: $0.005 \%$ acetic acid in 95:5 methanol:acetonitrile (vol:vol). Initial concentration of solvent B was $30 \%$, this was held for $30 \mathrm{~s}$, ramped to $45 \%$ over $30 \mathrm{~s}$, followed by a ramp to $75 \%$ B over the next 11 min. B was then ramped to $95 \%$ over $30 \mathrm{~s}$, held at $95 \%$ B for $3.5 \mathrm{~min}$, dropped back to 30 $\%$ over $30 \mathrm{~s}$ and then re-equilibrated at $30 \% \mathrm{~B}$ for $5.5 \mathrm{~min}$ before the next injection. The HPLC was interfaced to an Applied Biosystems/SCIEX Q-TRAP 5500 mass spectrometer with electrospray interface operated in the negative mode with the following settings: source voltage $-4,000 \mathrm{kV}$, GS1 40, GS2 40, CUR 40, TEM 450 and CAD gas HIGH.

Individual compounds were monitored with multiple reactions monitoring (MRM). Optimal instrument MRM parameters were obtained for the available standards using direct infusion. Data were acquired and analyzed using Analyst 1.5.1 and Multiquant 2.1.1 software, respectively. The standard curves were from 0 to 2,000 pg per sample and the limit of quantification was $50 \mathrm{pg}$.

Oxidative stress was also assessed by measuring 3-nitrotyrosine using the OxiSelect ${ }^{\mathrm{TM}}$ Nitrotyrosine ELISA Kit (Cell Biolabs, Inc, San Diego, CA, USA).

\section{Study protocol}

All except the sham dogs were assigned randomly to one of three groups at the time of initial surgery: (a) placebo, (b) metoprolol treatment, or (c) carvedilol treatment. All animals underwent weekly 2DE to evaluate for development of LV systolic dysfunction. Severe LV dysfunction occurred $5.8 \pm 0.8$ weeks after operation. At this time a 3 month treatment protocol was initiated: non-sham animals were treated orally with either placebo, metoprolol (12.5 mg BID for month 1, $25 \mathrm{mg}$ BID for month 2, and $50 \mathrm{mg}$ BID for month 3), or carvedilol (3.125 mg orally BID for month $1,6.25 \mathrm{mg}$ BID for month 2, and $12.5 \mathrm{mg}$ BID for month 3 ). These doses were selected to be comparable to an average human that is twice the weight of the dogs used.

At baseline and the end of each month of treatment, hemodynamic data were collected, 2DE was performed, and intra-myocardial fluid and venous blood were collected with the dogs anesthetized and intubated. Anesthesia was induced with intravenous fentanyl $\left(20 \mathrm{mg} \mathrm{kg}^{-1}\right)$, etomidate $\left(400 \mathrm{mg} \mathrm{kg}^{-1}\right)$, and diazepam $\left(300 \mathrm{mg} \mathrm{kg}^{-1}\right)$; and it was maintained with etomidate $\left(1 \mathrm{mg} \mathrm{min}^{-1}\right)$. During the same examination stress was induced by administering dobutamine (20-30 $\left.\mathrm{g} \mathrm{kg} \mathrm{min}^{-1}\right)$ intravenously at baseline and at month 3 . If target heart rate of $160-170 \mathrm{bpm}$ was not achieved, intravenous atropine $(0.5 \mathrm{mg})$ was also administered. At the conclusion of 3 months of therapy the animal was euthanized and the heart was removed for radiolabeled microsphere-derived blood flow analysis, histopathology, immunohistochemistry and oxidative stress analyses.

\section{Statistical methods}

Inter-stage comparisons were made using repeated measures ANOVA. When significance was found by ANOVA, individual comparisons between two stages were performed using student's $t$ test, with Bonferroni correction. Differences between stages were considered significant at $p<0.05$ (two-sided).

\section{Results}

Other than the seven sham dogs, 12 each were randomly assigned to the three treatment groups out of which three died before and two after start of treatment in the placebo group, one before start of treatment in the metoprolol group and two before start of treatment in the 
carvedilol group. No dog died after start of treatment in the two latter groups. Consequently the results reported here pertain to the 7 sham dogs and 8,11, and 10 dogs in the placebo, metoprolol, and carvedilol groups, respectively. As stated above, all measurements were made with the closed chest animals anesthetized and intubated.

\section{Hemodynamics}

Resting heart rate was not different between any of the groups between pre-surgery, baseline (development of severe LV dysfunction), and 1-3 months after treatment. Table 1 lists data at baseline and 3 months later. The mean aortic pressure at rest (measured in $\mathrm{mm} \mathrm{Hg}$ via indwelling catheter) was also not different at baseline between the groups. However, it was higher at the end of 3 months in the placebo compared to other groups. It was also higher in the placebo group compared to baseline. The rest double product, a measure of myocardial $\mathrm{O}_{2}$ consumption, was not different among the groups at baseline, but was lower in the sham and metoprolol groups at 3 months.

During stress, the heart rate increased equally among all groups at both baseline and 3 months. The mean aortic pressure also increase in all groups during stress but at 3 months this increase was higher in the placebo compared to the other groups. Similarly, the double product increased during stress in all groups without a significant difference between the groups at both baseline and 3 months.

\section{Global and regional function}

Both end-diastolic and end-systolic dimensions increased more between baseline and 3 months in the placebo compared to the treatment groups although this did not reach statistical significance (Fig. 1a). In comparison, while endsystolic wall stress increased in the placebo and metoprolol groups, it decreased significantly in the carvedilol group.

As expected, \%WT at rest did not change in the sham group after surgery, but decreased markedly and equally in all the three treatment groups (Table 2). In the placebo and metoprolol groups \%WT remained depressed for the entire 3 months of treatment while for the carvedilol group it improved every month compared to the placebo group. Only the carvedilol group showed a significant increase in resting \%WT between baseline and 3 months and by 3 months resting \%WT was higher than in the metoprolol group.

Stress was performed at baseline and 3 months where \%WT increased in all groups but was significantly lower in the treatment groups compared to the sham group ( $p<0.001$, Fig. 1b). At 3 months the stress \%WT remained unchanged in all groups compared to baseline except the carvedilol group where it was significantly higher ( $40 \pm 7$ vs. $34 \pm 7 \%, p=0.004)$ and where, unlike the other groups, it reached the level of the sham group $(43 \pm 1 \%)$.

\section{Regional MBF}

Resting MBF was measured at baseline (prior to starting any treatment) and at 3 months after completion of treatment (Table 3). At baseline, whereas transmural MBF did not decrease appreciably in any group, the endocardial MBF and the endocardial/epicardial MBF ratio were the lowest in the carvedilol group being significantly lower than the sham and metoprolol groups. At 3 months the endocardial MBF in the carvedilol group had improved significantly compared to baseline and had reached the same level as in the other groups. Similar results were seen for the endocardial/epicardial MBF ratio. Therefore, similar to \%WT, the carvedilol group showed the most improvement in resting MBF by 3 months, albeit starting from a lower value at baseline. 
At baseline MBF increased in all groups during stress showing the presence of MBF reserve (Table 4). The results were similar at 3 months in all groups except the sham group that showed further enhancement in flow reserve compared to the other three groups. The endocardial/epicardial MBF ratio improved marginally $(p=0.10)$ at 3 months in the carvedilol group whereas it remained unchanged in the other three groups.

\section{Interstitial leukocyte count and plasma cytokine levels}

Table 5 depicts the interstitial fluid leukocyte count. At baseline there was severe leukocytosis in the all groups except the sham group. The leukocyte count in the carvedilol group was significantly lower than the metoprolol group at 1 month after starting treatment. However, by 2 and 3 months, although still significantly lower than placebo, the leukocyte count in both BB treatment groups was similar to each other.

Myocardial interstitial fluid levels of TNF-a and IL-6 increased at 1 month in the placebo compared to the other groups, but declined to the same level as the other groups by 3 months (data not shown). In comparison, the levels of the pro-inflammatory IL-1 $\beta$ were lower at 3 months in the metoprolol and carvedilol compared to the placebo group (Fig. 2a). Conversely, the levels of the anti-inflammatory IL-10 declined by approximately half by 3 months in the placebo group, remained virtually unchanged in the metoprolol group, and increased 1.5-fold in the carvedilol group (Fig. 2b). None of the cytokines increased in the plasma at any stage and there was no correlation between plasma and myocardial interstitial fluid cytokine levels.

\section{Histopathology}

TTC staining showed no infarction in any of the animals in any group. The number of apoptotic cells per HPF was similar in each group $(5 \pm 2,7 \pm 3,6 \pm 3$ for placebo, metoprolol, and carvedilol, respectively) except for sham animals where there was no apoptosis. There was less fibrosis seen in the treatment groups compared to placebo with the carvedilol group showing significant reduction in fibrosis compared to the placebo group (Fig. 3a). Panel a in Fig. 4 illustrates an example of Masson's trichrome staining of tissue from each of the four groups with less generalized fibrosis in the carvedilol group.

Panel $b$ in Fig. 4 shows examples of capillaries in tissue from each group stained by von Willebrand factor. There is capillary rarefaction in the placebo and metoprolol groups and the capillary density appears to be higher in the carvedilol group compared to these two groups. Figure $3 \mathrm{~b}$ illustrates the capillary density in quantitative terms from all animals combined and the carvedilol group demonstrates a greater capillary density than the metoprolol and placebo groups.

\section{Oxidative stress}

Table 6 illustrates the results of the oxidative stress analyses. The carvedilol group shows lower MDA levels compared to the placebo and metoprolol groups. The isoprostane levels, however, were similar between the three groups. In comparison, 3-nitrotyrosine was not detected in any of the samples, which may have been due to low sensitivity of the kit.

\section{Discussion}

Our investigation demonstrates three new major findings in a chronic canine model of multivessel ischemic cardiomyopathy. First, compared to metoprolol, carvedilol treatment was associated with better resting global and regional LV function and better regional LV function during stress. Second, carvedilol was associated with greater improvements in resting endocardial MBF, albeit from the lowest level at baseline, which was associated with 
a higher capillary density. Third, carvedilol treated animals had least inflammation as evidenced by least myocardial leukocytosis and fibrosis and highest myocardial levels of the anti-inflammatory cytokine IL-10. Taken together these results suggest that carvedilol has anti-inflammatory and pro-angiogenic properties that improve regional perfusion and function in chronic myocardial ischemia.

Most large clinical trials of BB in CHF have included patients with idiopathic dilated as well as ischemic cardiomyopathy. Furthermore, a significant proportion of patients with ischemic cardiomyopathy have a prior MI. Other ischemic cardiomyopathy patients may have some myocardial segments that exhibit hibernation and/or some that may exhibit repetitive stunning $[5,6,8,14,15]$. Hence, the mechanism of reduced resting function is frequently heterogeneous not only between patients but also in the same patient, which may, in part, explain the conflicting results as well as controversy regarding the superiority of carvedilol over metoprolol in CHF.

In this study, we investigated the effects of BB in a canine model of chronic ischemic cardiomyopathy not associated with MI that is akin to the clinical situation in many patients. This model causes a global cardiomyopathy via chronic multivessel stenosis that is commonly seen in the clinical setting, which is in contrast to the more common canine model of rapid pacing induced cardiomyopathy mimicking the rarer clinical tachycardiainduced cardiomyopathy. In this model of regional (and as a consequence global) LV dysfunction we found carvedilol to be superior to metoprolol independent of beta blockade (no differences between treatment groups in stress-induced double product although these were measured under anesthesia). The primary basis for the putative superiority of carvedilol may lie with its anti-inflammatory and pro-angiogenic effects.

A unique strength of our study was the direct in vivo measurements of the components of myocardial interstitial space over time rather than relying on a single post-mortem analysis. In this manner, we noted that the attenuation of myocardial leukocytosis was more pronounced with carvedilol compared to metoprolol. The same can be stated for measuring the pro- and anti-inflammatory cytokines. Peripheral blood levels of these molecules were not related in any way to their myocardial levels, further emphasizing the importance of measurements made directly from the myocardium to assess drug effect.

We found that the pro-inflammatory cytokine IL-1 $\beta$ decreased significantly with both BB's. This cytokine has been implicated in heart failure by stimulation of hypertrophy and apoptosis as well as by decreasing collagen synthesis and increasing matrix metalloproteinase activity [1, 41]. It also inhibits phospholamban gene expression and intracellular calcium transients, among other detrimental effects [26]. Reduction of IL- $1 \beta$ in conjunction with attenuation of leukocytosis, confirms the previously described antiinflammatory properties of BB's [23].

In animals receiving carvedilol the anti-inflammatory cytokine IL-10 increased whereas in animals receiving metoprolol it did not change. To our knowledge this is the first study to describe these findings in a model of chronic ischemic cardiomyopathy not caused by MI. IL-10 is the most potent anti-inflammatory cytokine known, and inhibits monocyte/ macrophage-derived inflammatory cytokines such as TNF-a, IL-1, IL-6, IL-8, IL-12, granulocyte colony stimulating factor, MIP-1a, and MIP-2a [17]. It also inhibits CD14 and nuclear transfer factor and degrades mRNA for pro-inflammatory cytokines [4]. In addition, IL-10 suppresses cell surface expression of TNF receptors and promotes their shedding into the systemic circulation [10]. In addition to reduced leukocytosis we also found reduced fibrosis caused by carvedilol, which may be related to the increased levels of IL-10, although both BB demonstrated reduced fibrosis. 
In addition to cytokines, we examined the effect of the drugs on lipid peroxidation. MDA, a marker of oxidative stress, level was highest in the placebo group which was consistent with elevated MDA levels in patients with CHF [35, 38] and myocardial ischemia [32]. Oxidative stress was attenuated with carvedilol treatment, a finding also observed in patients with $\mathrm{CHF}$ [9]. Furthermore, in a study of smooth muscle cells exposed to elevated pressure, carvedilol but not propranolol, resulted in a reduction of oxidative stress [43].

\section{Study limitations}

All our measurements were performed with the animals anesthetized. Our hemodynamic data, therefore, were not acquired under physiological conditions. However, the method of anesthesia was similar in all animals. Despite randomization the carvedilol group had the lowest resting MBF. It is possible that more myocardial ischemia in this group resulted in greater collateral development and a consequent increase in resting MBF. However, these animals also showed the greatest attenuation of inflammation and the greatest increase in stress-induced \%WT. Therefore, drug effect is more likely than collateral growth to explain the results.

We used a fixed dose of the drugs and although the degree of beta blockade seemed equivalent in the carvedilol and metoprolol groups as assessed by heart rate and double product, it is always difficult to find equivalency between groups with fixed drug doses. However, we used comparable it not more stringent doses between the two BB's than used in previous clinical trials. At each month of treatment, the metoprolol to carvedilol dose ratio was 4:1, which was similar to a previous clinical trial [22] and more rigorous than that used in the COMET Trial [36] where the ratio was 2:1, and yet we found better myocardial regional function and perfusion in the carvedilol compared to the metoprolol group.

Nonetheless, our study was not designed as a pharmacological study to determine the optimal dose of the two drugs or to understand their pharmacokinetics. Our results, therefore, are solely pertinent to the doses of the two BB's used in this study.

The limited number of animals in each group precluded the assessment of the dose-response in terms of the variables we measured. In addition, we used short acting (tartrate) instead of the long acting (succinate) formulation of metoprolol. It would be interesting to know whether the results would be similar with the long acting preparation. Our results reflect follow-up studies after 3 months of treatment. It is possible that had we extended the followup period to 6 or 12 months, the effects of carvedilol and metoprolol would have become equivalent or the magnitude of change in the carvedilol group would have been even greater than metoprolol.

Finally, despite beta blockade, there was increase in heart rate and double product in both BB groups for which there could be several reasons. First is the use of anesthesia. Second, maximal beta blockade was probably not achieved, which anyway is not the purpose in treating heart failure. Third, both metoprolol and carvedilol are reversible beta-adrenoceptor antagonists, and dobutamine used could have displaced the antagonists. Fourth, it has been shown in a human cardiac tissue that carvedilol blocks $\beta 2$ - more than $\beta 1$-adrenoceptors [28], thus leaving the $\beta 1$-adrenoceptors available for activation by dobutamine.

\section{Conclusions}

In a canine model of chronic ischemic LV dysfunction we found carvedilol to be superior to metoprolol in improving resting perfusion and resting and stress-induced function. This improvement was related to the superior anti-inflammatory and pro-angiogenic properties of carvedilol. Our results are pertinent to only the doses of the drugs used in this study. Further studies are required to determine whether similar results can be obtained in other forms of 
ischemic cardiomyopathy, including those caused by or associated with MI. Further studies could also establish comparison between carvedilol and metoprolol at different doses than used in this study. Finally, whether the advantages of carvedilol described herein will offer a mortality benefit over metoprolol in patients with CHF may depend on its underlying mechanism, a focus that needs further research.

\section{Acknowledgments}

The authors would like to thank Craig Goodman, B.S. for his expert technical assistance with the experiments. We are also grateful to Melissa Bevard, Helen Liu, and Yan Zhao for their assistance with the histology and immunohistochemistry work and to Jay Phillips, Robert Webber, Lisa Bleyle and Dennis Koop from the Bioanalytical Shared Resource/Pharmacokinetics Core University Shared Resources at OHSU for their assistance with the oxidative stress assessment.

Supported in part by grants from the National Institutes of Health (K-08-HL0742901, D. Elizabeth Le and R01HL660346, Sanjiv Kaul). The radiolabeled microspheres were provided by Dupont Pharmaceuticals, North Billerica, MA, and the ultrasound equipment was supplied by Philips Ultrasound, Andover, MA.

\section{References}

1. Abbate A, Van Tassell BW, Seropian IM, Toldo S, Robati R, Varma A, Salloum FN, Smithson L, Dinarello CA. Inter-leukin-1 modulation using a genetically engineered antibody prevents adverse cardiac remodeling following acute myocardial infarction in the mouse. Eur J Heart Fail. 2010; 12:319-322. doi:10.1093/eurjhf/hfq017. [PubMed: 20335350]

2. Bin JP, Wei K, Pelberg RA, Coggins M, Goodman NC, Kaul S. Relation between regional function and coronary blood flow reserve in multivessel coronary artery stenosis. Am J Physiol. 2000; 279:H3058-H3064.

3. Bin JP, Pelberg RA, Coggins MP, Wei K, Kaul S. Mechanism of Inducible regional dysfunction during dipyridamole Stress. Circulation. 2002; 106:112-117. doi: 10.1161/01.CIR00000202230839005. [PubMed: 12093779]

4. Brandtzaeg P, Osnes L, Ovstebo R, Joo GB, Westvik AB, Kierulf P. Net inflammatory capacity of human septic shock plasma evaluated by a monocyte-based target cell assay: identification of interleukin-10 as a major functional deactivator of human monocytes. J Exp Med. 1996; 184:51-60. doi:10.1084/jem.184.1.51. [PubMed: 8691149]

5. Braunwald E, Kloner RA. The stunned myocardium: prolonged, postischemic ventricular dysfunction. Circulation. 1982; 66:1146-1149. doi:10.1161/01.CIR.66.6.1146. [PubMed: 6754130]

6. Braunwald E, Rutherford JD. Reversible ischemic left ventricular dysfunction: evidence for the "hibernating myocardium". J Am Coll Cardiol. 1986; 8:1467-1470. doi:10.1016/ S0735-1097(86)80325-4. [PubMed: 3782649]

7. Bristow MR, Adams KF, Bauman JL, Feldman AM, Giles TD, Goldstein S, Mann DL, Talbert RL. The COMET trial. Congest Heart Fail. 2005; 11:39-47. doi:10.1111/j.1527-5299.2005.04076.x. [PubMed: 15722669]

8. Camici PG, Wijns W, Borgers M, De Silva R, Ferrari J, Knuuti J, Lammerstma AA, Liedtke AJ, Paternostro G, Vatner SF. Pathophysiological mechanisms of chronic reversible left ventricular dysfunction due to coronary artery disease (hibernating myocardium). Circulation. 1997; 96:32053214. doi:10.1161/01.CIR.96.9.3205. [PubMed: 9386194]

9. Castro P, Vukasovic JL, Chiong M, Diaz-Araya G, Alcaino H, Copaja M, Valenzuela R, Greig D, Perez O, Corbalan R, Lavandero S. Effects of carvedilol on oxidative stress and chronotropic response to exercise in patients with chronic heart failure. Eur J Heart Fail. 2005; 7:1033-1039. doi: 10.1016/j.ejheart.2004.11.009. [PubMed: 16227141]

10. Clarke CJ, Hales A, Hunt A, Foxwell BM. IL-10 mediated suppression of TNF-a production is independent of its ability to inhibit NF-kB activity. Eur J Immunol. 1998; 28:1719-1726. doi: 10.1002/(SICI)1521-4141(199805)28:05<1719:AID-IMMU1719>3.0.CO;2-Q. [PubMed: 9603479] 
11. Firoozan S, Wei K, Linka A, Skyba D, Goodman NC, Kaul S. A canine model of chronic ischemic cardiomyopathy: characterization of regional flow-function relations. Am J Physiol. 1999; 276:H446-H455. [PubMed: 9950844]

12. Fishbein MC, Meerbaum S, Rit J, Lando U, Kanmatsuse K, Mercier JC, Corday E, Ganz W. Early phase acute myocardial infarct size quantification: validation of the triphenyl tetrazolium chloride tissue enzyme staining technique. Am Heart J. 1981; 101:593-600. doi: 10.1016/0002-8703(81)90226-X. [PubMed: 6164281]

13. Guth ND, Heusch G, Seitelberger R, Ross J Jr. Mechanism of beneficial effect of beta-adrenergic blockade on exercise-induced myocardial ischemia in conscious dogs. Circ Res. 1987; 60:738746. doi:10.1161/01.RES.60.5.738. [PubMed: 3594748]

14. Heusch G, Schulz R, Rahimtoola H. Myocardial hibernation: a delicate balance. Am J Physiol Heart Circ Physiol. 2005; 288:H984-H999. doi:10.1152/ajpheart.01109.2004. [PubMed: 15563526]

15. Heusch G. The regional myocardial flow-function relationship: a framework for an understanding of acute ischemia, hibernation, stunning and coronary microembolization. Circ Res. 2013; 112:1535-1537. doi:10.1161/CIRCRESAHA.113.301446. [PubMed: 23743225]

16. Heymann MA, Payne BD, Hoffman JI, Rudolph AM. Blood flow measurements with radio-nuclide labeled particles. Prog Cardiovasc Dis. 1997; 20:52-79. doi:10.1016/S0033-0620(77) 80005-4.

17. Lalani I, Bhoi K, Ahmed AF. Interleukin-10: biology, role in inflammation and autoimmunity. Ann Allergy. 1979; 79:469-483. doi:10.1016/S1081-1206(10)63052-9.

18. Le DE, Pelberg RA, Leong-Poi H, Bin JP, Linden J, Kaul S. A1-receptor blockade: a novel approach for assessing myocardial viability in chronic ischemic cardiomyopathy. J Am Soc Echocardiogr. 2003; 16:764-769. doi:10.1016/S0894-7317(03)00214-1. [PubMed: 12835664]

19. Le DE, Powers ER, Bin JP, Leong-Poi H, Goodman NC, Kaul S. Transmyocardial revascularization ameliorates Ischemia by reversing paradoxical catecholamine-induced vasoconstriction. J Nucl Cardiol. 2007; 14:207-214. doi:10.1016/j.nuclcard.2006.12.328. [PubMed: 17386383]

20. Li B, Liao YH, Cheng X, Ge H, Guo H, Wang M. Effects of carvedilol on cardiac cytokines expression and remodeling in rat with acute myocardial infarction. Int J Cardiol. 2006; 111:247255. doi:10.1016/j.ijcard.2005.08.065. [PubMed: 16310260]

21. Libby P, Maroko PR, Covell JW, Malloch CI, Ross J Jr, Braunwald E. Effect of practolol on the extent of myocardial ischaemic injury after experimental coronary occlusion and its effect on ventricular function in the normal and ischaemic heart. Cardiovasc Res. 1973; 7:167-173. doi: 10.1093/cvr/7.2.167. [PubMed: 4144351]

22. Maack C, Elter T, Nickenig G, LaRosee K, Crivaro M, Stäblein A, Wuttke H, Böhm M. Prospective crossover comparison of carvedilol and metoprolol in patients with chronic heart failure. J Am Coll Cardiol. 2001; 38:939-946. doi:10.1016/S0735-1097(01)01471-1. [PubMed: 11583862]

23. Mann DL. Inflammatory mediators and the failing heart: past, present, and the foreseeable future. Circ Res. 2002; 91:988-998. doi:10.1161/01.RES.0000043825.01705.1B. [PubMed: 12456484]

24. Matsuzaki M, Patritti J, Tajimi T, Miller M, Kemper WS, Ross J. Effects of beta-blockade on regional myocardial blood flow and function during exercise. Am J Physiol. 1984; 247:H52-H60. [PubMed: 6146266]

25. McBride BF, White CM. Critical differences among beta-adrenoreceptor antagonists in myocardial failure: debating the MERIT of COMET. J Clin Pharmacol. 2005; 45:6-24. doi: 10.1177/0091270004269841. [PubMed: 15601801]

26. McTiernan CF, Lemster BH, Frye C, Brooks S, Combes A, Feldman AM, Charles F. Interleukin-1 $\beta$ inhibits phospholamban gene expression in cultured cardiomyocytes. Circ Res. 1997; 81:493-503. doi:10.1161/01.RES.81.4.493. [PubMed: 9314830]

27. MERIT-HF Study Group. Effect of metoprolol CR/XL in chronic heart failure: metoprolol CR/XL randomised intervention trial in-congestive heart failure (MERIT-HF). Lancet. 1999; 353:20012007. doi:10.1016/S0140-6736(99)04440-2. [PubMed: 10376614] 
28. Molenaar P, Christ T, Ravens U, Kaumann A. Carvedilol blocks beta2- more than beta1adrenoceptors in human heart. Cardiovas Res. 2006; 69:128-139. doi:10.1016/j.cardiores. 2005.08.024.

29. Moore K, Roberts LJ. Measurement of lipid peroxidation. Free Radic Res. 1998; 28:659-671. doi: 10.3109/10715769809065821. [PubMed: 9736317]

30. Oh BH, Ono S, Gilpin E, Ross J. Altered left ventricular remodeling with beta-adrenergic blockade and exercise after coronary reperfusion in rats. Circulation. 1993; 87:608-616. doi:10.1111/j. 1540-8191.1996.tb00073.x. [PubMed: 8093867]

31. Okafor CC, Perreault-Micale C, Hajjar RJ, Lebeche D, Skiroman K, Jabbour G, Doye AA, Lee MX, Laste N, Gwathmey JK. Chronic treatment with carvedilol improves ventricular function and reduces myocyte apoptosis in an animal model of heart failure. BMC Physiol. 2003; 3:1-9. doi: 10.1186/1472-6793-3-6. [PubMed: 12597777]

32. Oldroyd KG, Chopra M, Rankin AC, Belch JJF, Cobbe SM. Lipid peroxidation during myocardial ischaemia induced by pacing. Br Heart J. 1990; 63:88-92. doi:10.1136/hrt.63.2.88. [PubMed: 2317414]

33. Packer M, Bristow MR, Cohn JN, Colucci WS, Fowler MB, Gilbert EM, Shusterman NH. The effect of carvedilol on morbidity and mortality in patients with chronic heart failure. N Engl J Med. 1996; 334:1349-1355. doi:10.1056/NEJM199605233342101. [PubMed: 8614419]

34. Pelberg RA, Spotnitz WD, Bin J-P, Le E, Goodman NC, Kaul S. Mechanism of myocardial dysfunction in the presence of chronic coronary stenosis and normal resting myocardial blood flow: clinical implications. J Am Soc Echocardiogr. 2001; 14:1047-1056. doi:10.1067/mje. 2001.113232. [PubMed: 11696827]

35. Polidori MC, Savino K, Alunni G, Freddio M, Senin U, Sies H, Stahl W, Mecocci P. Plasma lipophilic antioxidants and malondialhehyde in congestive heart failure patients: relationship to disease severity. Free Radic Biol Med. 2002; 32:148-152. [PubMed: 11796203]

36. Poole-Wilson PA, Swedberg K, Cleland JGF, Di Lenarda A, Hanrath P, Komajda M, Lubsen J, Lutiger B, Metra M, Remme WJ, Torp-Pedersen C, Scherhag A, Skene A, For the COMET Investigators. Comparison of carvedilol and metoprolol on clinical outcomes in patients with chronic heart failure in the carvedilol or metoprolol European trial (COMET): randomized controlled trial. Lancet. 2003; 362:7-13. doi:10.1016/S0140-6736(03)13800-7. [PubMed: 12853193]

37. Prabhu SD, Chandrasekar B, Murray DR, Freeman GL. $\beta$-Adrenergic blockade in developing Heart failure. Effects on myocardial inflammatory cytokines, nitric oxide, and remodeling. Circulation. 2000; 101:2103-2109. doi:10.1161/01.CIR.101.17.2103.

38. Radovanovic S, Savic-Radojevic A, Pljesa-Ercegovac M, Djukic T, Suvakov S, Krotin M, Simic DY, Matic M, Radojicic Z, Pekmezovic T, Simic T. Markers of oxidative damage and antioxidant enzyme activities as predictors of morbidity and mortality in patients with chronic heart failure. J Card Fail. 2012; 18:493-501. doi:10.1016/j.cardfail.2012.04.003. [PubMed: 22633308]

39. Rowland DG, Gutgesell HP. Use of mean arterial pressure for noninvasive determination of left ventricular end-systolic wall stress in infants and children. Amer J Cardiol. 1994; 74:98-99. (1994). [PubMed: 8017319]

40. Ruwald MH, Ruwald AH, Jons C, Alexis A, McNitts S, Zareba W, Moss AJ. Effect of metoprolol versus carvedilol on outcomes in MADIT-CRT (multicenter automatic defibrillator implantation trial with cardiac resynchronization therapy. J Am Coll Cardiol. 2013; 61:1518-1526. [PubMed: 23500269]

41. Siwik DA, Chang DL, Colucci WS. Interleukin-1 $\beta$ and tumor necrosis factor-a decrease collagen synthesis and increase matrix metalloproteinase activity in cardiac fibroblasts in vitro. Circ Res. 2000; 86:1259-1265. doi:10.1161/01.RES.86.12.1259. [PubMed: 10864917]

42. Sklenar J, Jayaweera AR, Kaul S. A computer-aided approach for the quantification of regional left ventricular function using two-dimensional echocardiography. J Am Soc Echo-cardiogr. 1992; 5:33-40.

43. Yasunari K, Maeda K, Nakamura M, Yoshikawa J. Carvedilol inhibits pressure-induced increase in oxidative stress in coronary smooth muscle cells. Hypertens Res. 2002; 25:419-425. [PubMed: 12135321] 
A

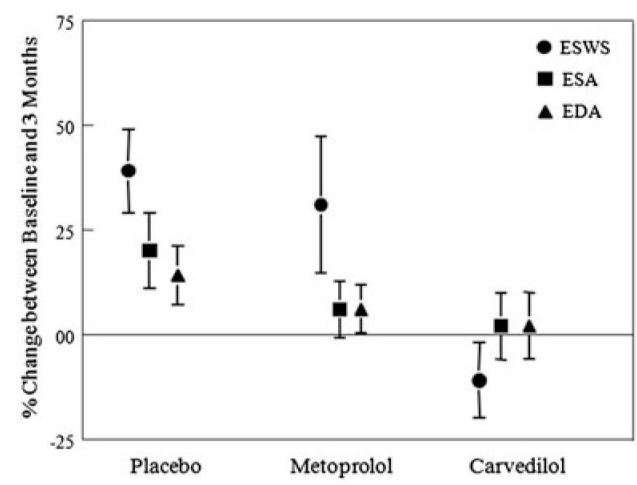

B $\square$ Sham $\square$ Placebo $\square$ Metoprolol $\square$ Carvedilol

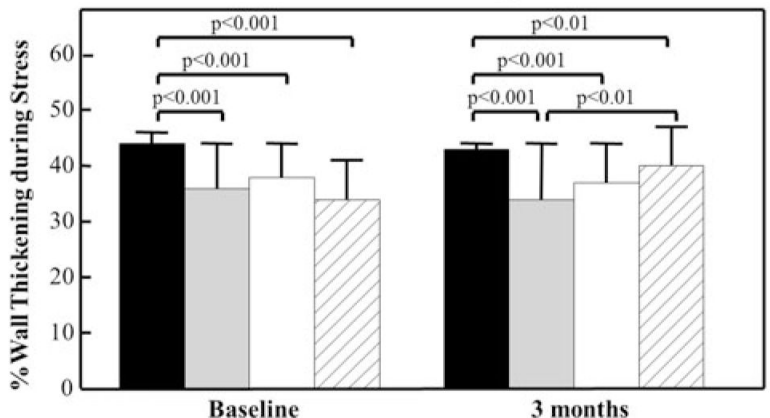

Fig. 1.

a Percent change between baseline and 3 months of endsystolic wall stress (ESWS), endsystolic area (ESA), and end-diastolic area (EDA) in the placebo, metoprolol, and carvedilol groups. Data are presented as mean \pm SEM. b \%WT in the sham, placebo, metoprolol, and carvedilol groups at baseline and 3 months during stress. Data are presented as mean \pm SEM 

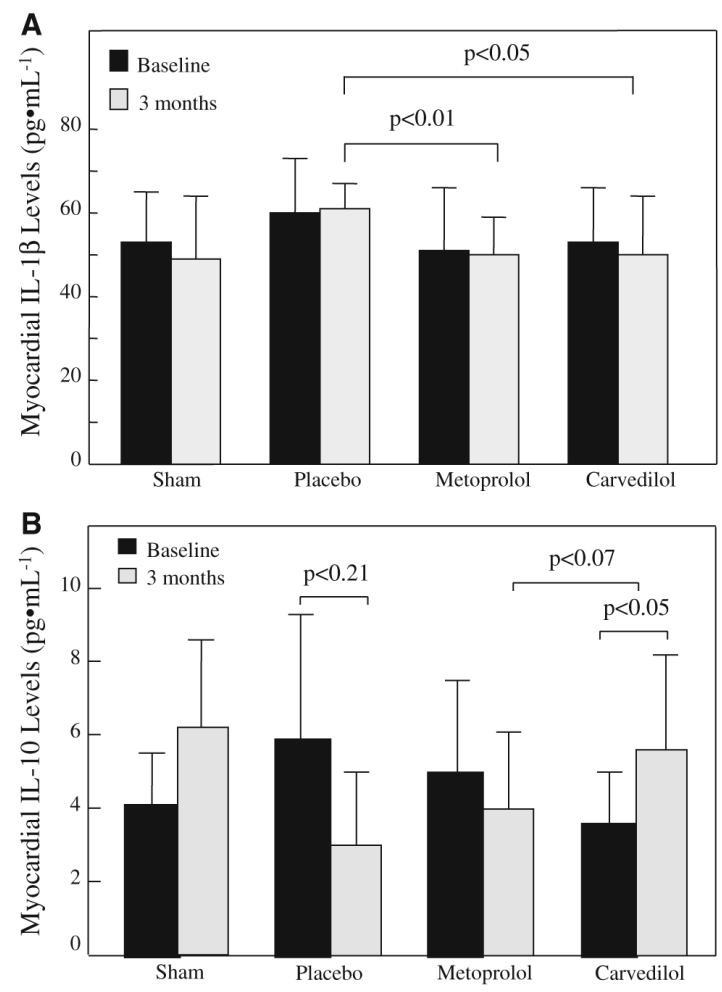

Fig. 2.

Myocardial levels of IL-1 $\beta$ (a) and IL-10 (b) in the sham, placebo, metoprolol, and carvedilol groups at baseline and 3 months. Data are presented as mean \pm SEM 

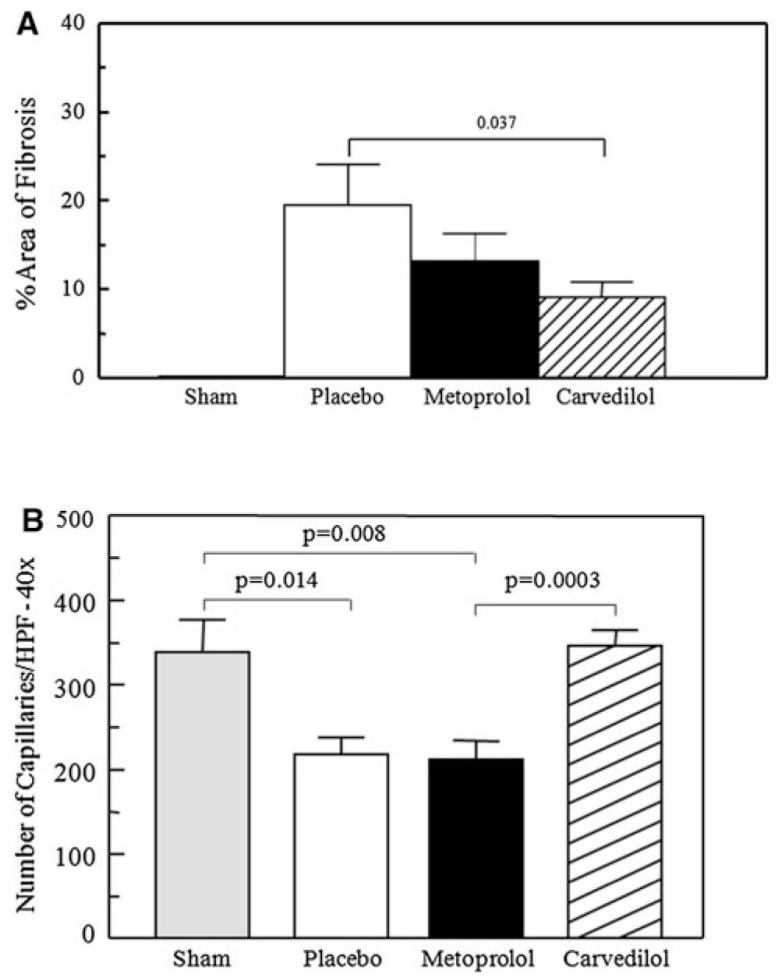

Fig. 3.

Quantitative analysis of histopathology data (a) fibrosis and (b) capillary density in the sham, placebo, metoprolol, and carvedilol groups. Data are presented as mean \pm SEM 


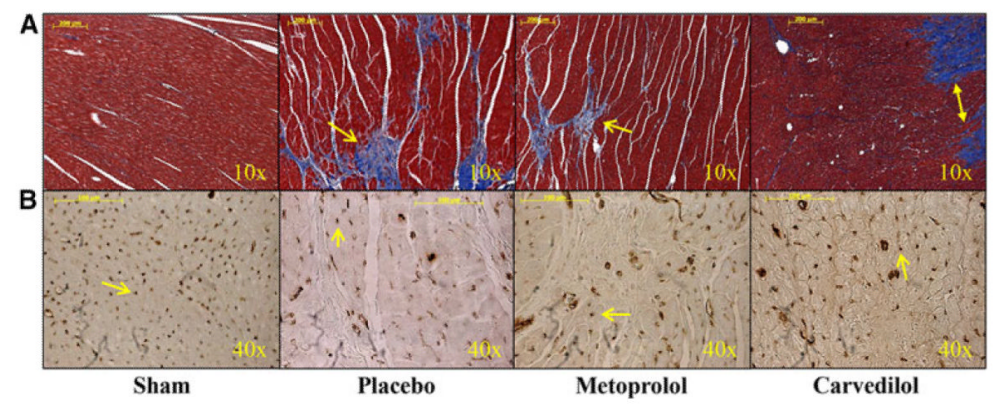

Fig. 4.

Representative histology images of fibrosis stained blue and identified by yellow arrow (a) at $\times 10$ magnification and capillaries stained brown and identified by black arrow (b) at $\times 40$ magnification 
Table 1

Hemodynamic data at baseline and at 3 months during rest and stress

\begin{tabular}{lccccl}
\hline Variable & Sham & Placebo & Metoprolol & Carvedilol & $\boldsymbol{p}$ value ANOVA \\
\hline Rest & & & & & \\
HR (baseline) & $61 \pm 8$ & $55 \pm 5$ & $54 \pm 2$ & $61 \pm 7$ & $\mathrm{~ns}$ \\
HR (3 months) & $55 \pm 7$ & $62 \pm 7$ & $54 \pm 2$ & $60 \pm 5$ & $\mathrm{~ns}$ \\
BP (baseline) & $73 \pm 5$ & $80 \pm 6$ & $81 \pm 3$ & $83 \pm 6$ & $\mathrm{~ns}$ \\
BP (3 months) & $77 \pm 4$ & $108 \pm 9$ & $84 \pm 4^{*}$ & $80 \pm 3^{\dagger}$ & $<0.05$ \\
DP (Baseline) & $52 \pm 4$ & $65 \pm 7$ & $68 \pm 6$ & $72 \pm 13$ & $\mathrm{~ns}$ \\
DP (3 months) & $60 \pm 5$ & $88 \pm 12$ & $68 \pm 3$ & $85 \pm 7 *$ & 0.03 \\
Stress & & & & & \\
HR (baseline) & $114 \pm 5$ & $108 \pm 7$ & $119 \pm 9$ & $112 \pm 9$ & $\mathrm{~ns}$ \\
HR (3 months) & $109 \pm 10$ & $119 \pm 11$ & $109 \pm 9$ & $113 \pm 10$ & $\mathrm{~ns}$ \\
BP (Baseline) & $115 \pm 5$ & $114 \pm 6$ & $116 \pm 7$ & $121 \pm 5$ & $\mathrm{~ns}$ \\
BP (3 months) & $128 \pm 6$ & $175 \pm 23$ & $131 \pm 7^{*}$ & $138 \pm 12$ & $<0.05$ \\
DP (Baseline) & $201 \pm 28$ & $193 \pm 14$ & $239 \pm 29$ & $208 \pm 22$ & $\mathrm{~ns}$ \\
DP (3 months) & $227 \pm 14$ & $283 \pm 34$ & $239 \pm 27$ & $251 \pm 37$ & $\mathrm{~ns}$ \\
\hline
\end{tabular}

Data are presented as mean \pm SEM

$H R$ heart rate, $B P$ mean aortic blood pressure, $D P$ double product/100

$p<0.05$ vs. placebo

${ }^{\dagger} p<0.01$ vs. placebo

${ }_{p}<0.05$ vs. metoprolol 


\section{Table 2}

Resting \%WT at baseline and 1, 2, and 3 months later

\begin{tabular}{lclclc}
\hline \%WT & Sham & Placebo & Metoprolol & Carvedilol & $\boldsymbol{p}$ value ANOVA \\
\hline Baseline & $37.0 \pm 0.4$ & $24.0 \pm 2$ & $27.3 \pm 1.3$ & $24.4 \pm 1.4$ & 0 \\
1 month & $37.5 \pm 0.4$ & $20.8 \pm 1.0$ & $25.7 \pm 1.0$ & $28.7 \pm 1.2^{*}$ & 0 \\
2 months & $37.3 \pm 0.4$ & $23.3 \pm 1.4$ & $27.7 \pm 1.0$ & $31.1 \pm 1.3^{*}$ & 0 \\
3 months & $37.1 \pm 0.4$ & $22.1 \pm 1.4$ & $28.4 \pm 1.3$ & $32.0 \pm 1.2^{* * *}$ & 0 \\
\hline
\end{tabular}

Data are presented as mean \pm SEM

$p<0.05$ vs. placebo

${ }^{\dagger} p<0.05$ vs. metoprolol

${ }_{p}^{+}<0.05$ vs. baseline 
Table 3

Myocardial ( $\mathrm{mL} \mathrm{min}^{-1} \mathrm{~g}^{-1}$ ) blood Flow at baseline and 3 months during rest

\begin{tabular}{lccccc}
\hline MBF $\left(\mathbf{m L ~} \mathbf{~ m i n}^{-\mathbf{1}} \mathbf{g}^{-\mathbf{1}}\right)$ & Sham & Placebo & Metoprolol & Carvedilol & $\boldsymbol{p}$ value ANOVA \\
\hline Baseline & & & & \\
Transmural MBF & $1.49 \pm 0.52$ & $1.18 \pm 0.74$ & $1.41 \pm 0.69$ & $0.83 \pm 0.54$ & 0.620 \\
Endocardial MBF & $2.59 \pm 0.52$ & $1.57 \pm 0.97$ & $1.98 \pm 0.91$ & $0.96 \pm 0.38^{\dagger \dagger}$ & 0.003 \\
Epicardial MBF & $1.04 \pm 0.48$ & $0.97 \pm 0.62$ & $1.12 \pm 0.70$ & $0.83 \pm 0.74$ & 0.660 \\
EER & $2.78 \pm 0.82$ & $1.73 \pm 0.59$ & $1.79 \pm 0.41$ & $1.48 \pm 0.54^{\dagger}$ & 0.001 \\
3 months & & & & \\
Transmural MBF & $1.31 \pm 0.57$ & $1.42 \pm 0.54$ & $1.32 \pm 0.60$ & $1.29 \pm 0.73$ & 0.97 \\
Endocardial MBF & $2.27 \pm 0.88$ & $2.13 \pm 0.28$ & $2.02 \pm 0.82$ & $1.81 \pm 1.04^{*}$ & 0.73 \\
Epicardial MBF & $1.10 \pm 0.53$ & $1.12 \pm 0.42$ & $1.04 \pm 0.57$ & $0.99 \pm 0.57$ & 0.96 \\
EER & $2.17 \pm 0.32$ & $1.95 \pm 0.60$ & $2.09 \pm 0.56$ & $1.84 \pm 0.37$ & 0.45 \\
\hline
\end{tabular}

EER Endocardial/epicardial MBF ratio

${ }^{*}<0.05$ vs. baseline

${ }^{\dagger} p<0.01$ vs. sham

${ }^{\ddagger} p<0.001$ vs. metoprolol at baseline 
Table 4

Myocardial blood flow $\left(\mathrm{mL} \mathrm{min}^{-1} \mathrm{~g}^{-1}\right)$ at baseline and 3 months during stress

\begin{tabular}{lcllll}
\hline MBF & Sham & Placebo & Metoprolol & Carvedilol & $p$ value \\
\hline Baseline & & & & & \\
Transmural MBF & $4.11 \pm 0.62$ & $2.81 \pm 1.90$ & $4.31 \pm 1.72$ & $3.79 \pm 1.10$ & 0.079 \\
Endocardial MBF & $6.15 \pm 0.92$ & $3.40 \pm 1.97$ & $5.03 \pm 2.28$ & $4.14 \pm 1.66$ & 0.098 \\
Epicardial MBF & $3.61 \pm 0.31$ & $2.64 \pm 2.01$ & $4.14 \pm 1.61$ & $3.72 \pm 0.85$ & 0.082 \\
EER & $1.71 \pm 0.24$ & $1.59 \pm 0.64$ & $1.30 \pm 0.49$ & $1.09 \pm 0.32$ & 0.111 \\
3 months & & & & & \\
Transmural MBF & $5.34 \pm 2.03$ & $2.77 \pm 2.33^{*}$ & $4.29 \pm 1.70$ & $3.31 \pm 0.65^{\dagger}$ & 0.017 \\
Endocardial MBF & $7.24 \pm 2.74$ & $3.05 \pm 2.61^{\dagger}$ & $5.03 \pm 2.03^{* \dagger}$ & $4.06 \pm 1.47^{\dagger}$ & 0.002 \\
Epicardial MBF & $4.47 \pm 1.72$ & $2.69 \pm 2.38$ & $4.13 \pm 1.77$ & $2.98 \pm 0.60^{\#}$ & 0.075 \\
EER & $1.66 \pm 0.29$ & $1.55 \pm 0.75$ & $1.30 \pm 0.45$ & $1.37 \pm 0.46$ & 0.400 \\
\hline
\end{tabular}

ANOVA analysis of variance, EER endocardial/epicardial MBF ratio

$*$

$p<0.05$ vs. sham

${ }^{\dagger} p<0.01$ vs. sham

${ }^{\ddagger} p<0.05$ vs. placebo

${ }^{\#} p<0.05$ vs. baseline 
Table 5

Interstitial fluid leukocyte count $\left(\mu \mathrm{L}^{-1}\right)$

\begin{tabular}{llcccc}
\hline Time & Sham $(\boldsymbol{n}=7)$ & Placebo $(\boldsymbol{n}=8)$ & Metoprolol $(\boldsymbol{n}=\mathbf{1 1})$ & Carvedilol $(\boldsymbol{n}=10)$ & $\boldsymbol{p}$ value ANOVA \\
\hline Baseline & $5 \pm 2$ & $16 \pm 6$ & $12 \pm 3$ & $20 \pm 8$ & 0.84 \\
1 month & $5 \pm 2^{\dagger \S}$ & $126 \pm 55$ & $28 \pm 8^{*}$ & $9 \pm 2^{\dagger \dagger}$ & 0.002 \\
2 months & $7 \pm 2^{*}$ & $118 \pm 64$ & $8 \pm 7^{*}$ & $11 \pm 3^{*}$ & 0.009 \\
3 months & $3 \pm 1^{\dagger}$ & $65 \pm 21$ & $9 \pm 4^{*}$ & $8 \pm 2^{\dagger}$ & 0 \\
\hline
\end{tabular}

Data are presented as mean \pm SEM

* $p<0.05$ vs. placebo

${ }^{\dagger} p<0.01$ vs. placebo

${ }^{+} p<0.05$ vs. metoprolol

$\S_{p<0.01 \text { vs. metoprolol }}$ 
Table 6

Results of oxidative stress measurements

\begin{tabular}{|c|c|c|c|}
\hline Variable & Placebo & Metoprolol & Carvedilol \\
\hline MDA (nM mg protein $\left.{ }^{-1}\right)$ & $3.11 \pm 0.37$ & $2.66 \pm 0.33$ & $2.13 \pm 0.14^{*}$ \\
\hline Total isoP III or 8-iso- $\mathrm{PGF}_{2 a}\left(\mathrm{pg}\right.$ mg protein $\left.{ }^{-1}\right)$ & $3,958 \pm 389$ & $3,954 \pm 322$ & $3,455 \pm 313$ \\
\hline Total isoP VI or $5-\mathrm{F}_{2}$-isoP (pg mg protein ${ }^{-1}$ ) & $14,300 \pm 1,424$ & $15,629 \pm 1,378$ & $13,993 \pm 1,083$ \\
\hline
\end{tabular}

Data are presented as mean \pm SEM

$M D A$ malondialdehyde, IsoP isoprostane

${ }^{*} p<0.05$ vs. placebo 